\title{
Veno-Arterial Extracorporeal Membrane Oxygenation with Conventional Anticoagulation Can Be a Best Solution for Shock Due to Massive PE
}

\author{
Yusuke Watanabe, ${ }^{1}$ MD, Kenichi Sakakura, ${ }^{1} \mathrm{MD}$, Naoyuki Akashi, ${ }^{1} \mathrm{MD}$, Mami Ishikawa, ${ }^{2} \mathrm{MD}$, \\ Yousuke Taniguchi, ${ }^{1}$ MD, Kei Yamamoto, ${ }^{1}$ MD, Hiroshi Wada, ${ }^{1}$ MD, \\ Hideo Fujita, ${ }^{1}$ MD and Shin-ichi Momomura, ${ }^{1}$ MD
}

\begin{abstract}
Summary
While most of pulmonary thromboembolism (PE) cases can be managed by thrombolytic and anticoagulation therapy, massive PE remains a life-threatening disease. Although surgical embolectomy can be a curative therapy for massive PE, peri-operative mortality for hemodynamically collapsed PE is extremely high. We present a case of hemodynamically collapsed massive PE. We avoided either thrombolytic therapy or surgical embolectomy, because the patient had recent cerebral contusion. Therefore, we managed the patient with the combination of veno-arterial extracorporeal membrane oxygenation (V-A ECMO) and conventional anticoagulation, which dramatically improved the patient's hemodynamics. In conclusion, the combination of V-A ECMO and conventional anticoagulation may be the preferred first line therapy for the patients with cardiogenic shock following massive PE.
\end{abstract}

(Int Heart J 2017; 58: 831-834)

Key words: Massive pulmonary embolism, Cardiogenic shock, Traumatic subarachnoid hemorrhage

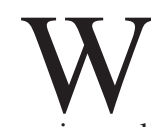

hile most of pulmonary thromboembolism (PE) cases can be managed by thrombolytic and anticoagulation therapy, massive PE remains a life-threatening disease. ${ }^{1-3)}$ Surgical embolectomy can be a curative therapy for massive PE. ${ }^{4}$ However, perioperative mortality for hemodynamically collapsed PE ranges from $43 \%$ to $84 \%{ }^{5)}$ Therefore, when we encounter a hemodynamically collapsed massive $\mathrm{PE}$, we have to make a difficult decision whether we should perform surgical embolectomy in a short time.

Veno-arterial extracorporeal membrane oxygenation (V-A ECMO) plays an important role in managing cardiogenic shock including massive PE. ${ }^{\text {) }}$ In this case report, we present a case of hemodynamically collapsed massive PE. Surgical embolectomy seemed to be needed to recover from shock, but the surgical risk was extremely high because the patient had a recent cerebral contusion. V-A ECMO stabilized hemodynamics, and helped us to make a decision regarding treatment strategy.

\section{Case Report}

A 55 year-old male patient with severe traumatic subarachnoid hemorrhage and hemorrhagic cerebral contusion was admitted to the intensive care unit (ICU) of our hospital. He had been immobilized in the ICU for 2 weeks. Although he put on a foot pump device to avoid deep vein thrombosis, he could not receive anticoagulation therapy because of the concerns of worsening brain hemorrhage. He started walking with parallel bars on day 28 . At day 33, when he underwent rehabilitation program as usual, he suddenly fell down. His Glasgow coma scale was E4 V1 M5. His blood pressure (BP) and heart rate were $74 / 47 \mathrm{mmHg}$ and $116 / \mathrm{min}$, respectively. His respiratory rate was 30 breaths/min, and showed hypoxia $\left(\mathrm{SpO}_{2}\right.$ $75 \%$ ). An electrocardiogram (ECG) revealed sinus tachycardia, right axis deviation, and a right bundle branch block (Figure 1). Contrast enhanced computed tomography (CT) showed massive pulmonary thrombi in bilateral main trunks of the pulmonary artery (Figure $2 \mathrm{~A}$ ). We diagnosed him as massive PE. Then, he developed pulseless electrical activity following CT examination. We started cardio-pulmonary resuscitation, and inserted V-A ECMO. His BP recovered to $79 / 66 \mathrm{mmHg}$ using V-A ECMO and catecholamine support when he readmitted to the coronary care unit (CCU) of our hospital. His pulse pressure (PP) was only $13 \mathrm{mmHg}$, which suggests the severely decreased cardiac output. We did not try to insert a pulmonary artery catheter, because of massive thrombus in pulmonary arteries.

We could not perform thrombolysis because of a recent episode of intracranial hemorrhage. Although surgical

From the ${ }^{1}$ Department of Cardiovascular Medicine, Saitama Medical Center, Jichi Medical University, Saitama, Japan and ${ }^{2}$ Department of Neurosurgery, Saitama Medical Center, Jichi Medical University, Saitama, Japan.

Address for correspondence: Kenichi Sakakura, MD, Department of Cardiovascular Medicine, Saitama Medical Center, Jichi Medical University, 1-847 Amanuma, Omiya-ku, Saitama, Saitama 330-8503 Japan. E-mail: ksakakura@jichi.ac.jp

Received for publication September 26, 2016. Revised and accepted December 20, 2016.

Released in advance online on J-STAGE September 30, 2017.

doi: 10.1536/ihj.16-483

All rights reserved by the International Heart Journal Association. 


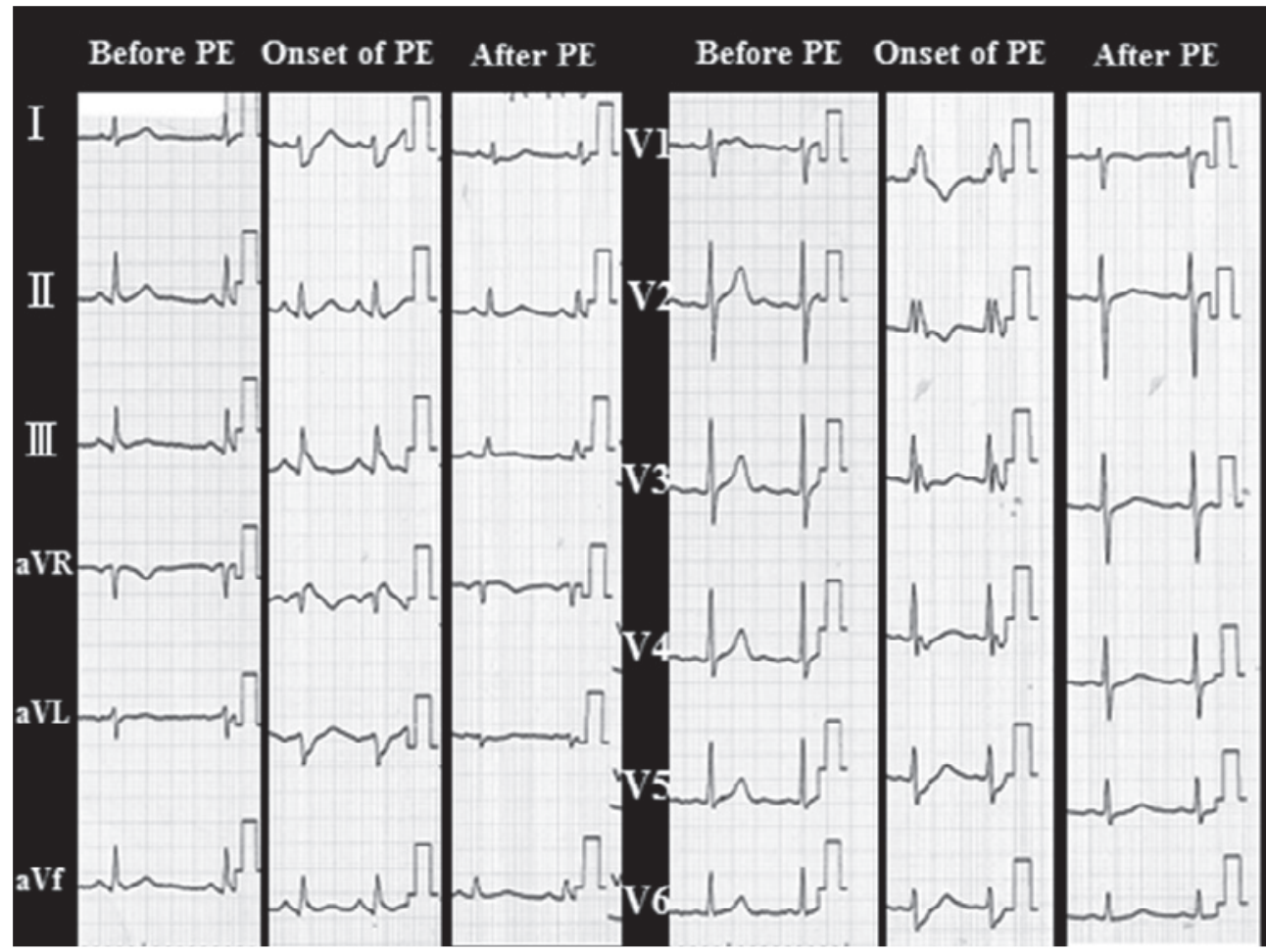

Figure 1. Electrocardiograms (ECG) of before PE, at the onset of PE, and after PE (day 2) were shown in the left, center, and right, respectively. ECG at the onset of PE showed sinus tachycardia (heart rate 132/minute), right axis deviation and complete right bundle branch block (CRBBB), while ECGs before PE and after PE did not show either right axis deviation or CRBBB.

embolectomy was considered as a treatment option, it requires a large amount of anticoagulation, which may trigger the relapse of intracranial hemorrhage during surgery. Therefore, we hesitated to perform emergent surgical embolectomy. We decided to treat him with conventional anticoagulation (unfractionated heparin) and V-A ECMO for 24-48 hours and reconsider surgical embolectomy if the initial treatment failed. Hemodynamic status and respiratory condition drastically improved in 48 hours (Figure 3 ). PP increased from $22 \mathrm{mmHg}$ to $35 \mathrm{mmHg}$ in 24 hours, and increased to $43 \mathrm{mmHg}$ in 48 hours, which suggests the recovery of his cardiac output. V-A ECMO was removed without any complications at 72 hours. The patient was extubated and woke up without additional neurological abnormality at 102 hours. Follow up CT images showed that pulmonary embolism in main pulmonary artery gradually reduced (Figure 2B-D). The ECG showed normal sinus rhythm and improvement of right axis deviation and CRBBB (Figure 1). The patient restarted rehabilitation, and we switched heparin to warfarin. He transferred to the rehabilitation hospital at day 19 from the onset of PE.

\section{Discussion}

The present case suggests that V-A ECMO with conventional anticoagulation can be a curative therapy like surgical embolectomy or thrombolysis using t-PA. As compared to surgical embolectomy or thrombolysis, V-A
ECMO with conventional anticoagulation is the least invasive strategy, which would be important for patients with comorbidity such as recent stroke or trauma. Furthermore, if V-A ECMO with conventional anticoagulation would not improve patient's hemodynamics, we could reconsider to perform surgical embolectomy as long as V-A ECMO can work. Therefore, V-A ECMO with conventional anticoagulation may be the preferred first line therapy not only for the patients with contraindication to thrombolysis, but also for any patients who developed cardiogenic shock following massive PE.

The reason why V-A ECMO with conventional anticoagulation worked for massive PE should be discussed. $\mathrm{PE}$ is currently classified as massive PE, submassive PE, or nonmassive PE according to the hemodynamic instability. ${ }^{7)}$ Therefore, thrombus burden in a massive PE is not necessarily greater than that in a submassive PE. ${ }^{7)}$ Because stabilizing hemodynamics should be more important than reducing thrombus burden in the treatment of massive $\mathrm{PE}{ }^{8)}$ prompt insertion of $\mathrm{V}-\mathrm{E}$ ECMO would have priority over thrombolysis. Furthermore, conventional anticoagulation can also reduce thrombus burden in PE or deep venous thrombosis. ${ }^{9-11)}$ If conventional anticoagulation could reduce even a little of thrombus burden under V-A ECMO support, hemodynamics in massive $\mathrm{PE}$ may improve in a short time. Moreover, PP was the useful indicator for evaluating hemodynamics, because we could not use a pulmonary artery catheter due to massive thrombus in pulmonary arteries. 

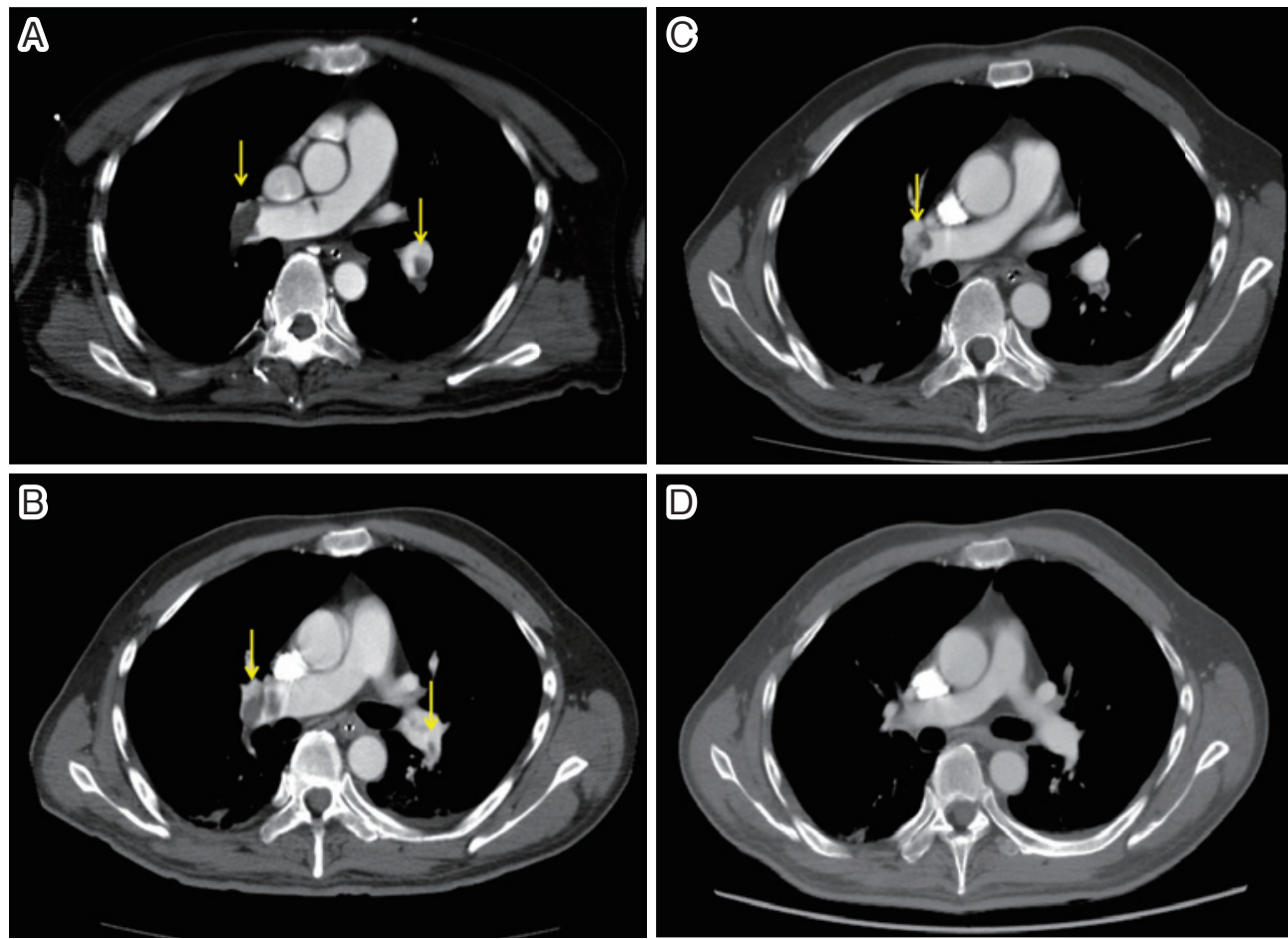

Figure 2. A: The Computed tomography (CT) image showed pulmonary embolism in bilateral main pulmonary trunk (arrows) (onset of PE). B: The CT image of day 4 from the onset of PE. Thrombus in bilateral main pulmonary trunk (arrows) remained visible, but the amount of thrombus decreased. C: The CT image of day 12 from the onset of PE. Thrombus in right main pulmonary trunk (arrow) is still visible, but there is no thrombus in left main pulmonary trunk. D: The CT image of day 16 from the onset of PE. There was no visible thrombus in bilateral main pulmonary trunks.

\section{Clinical Course}

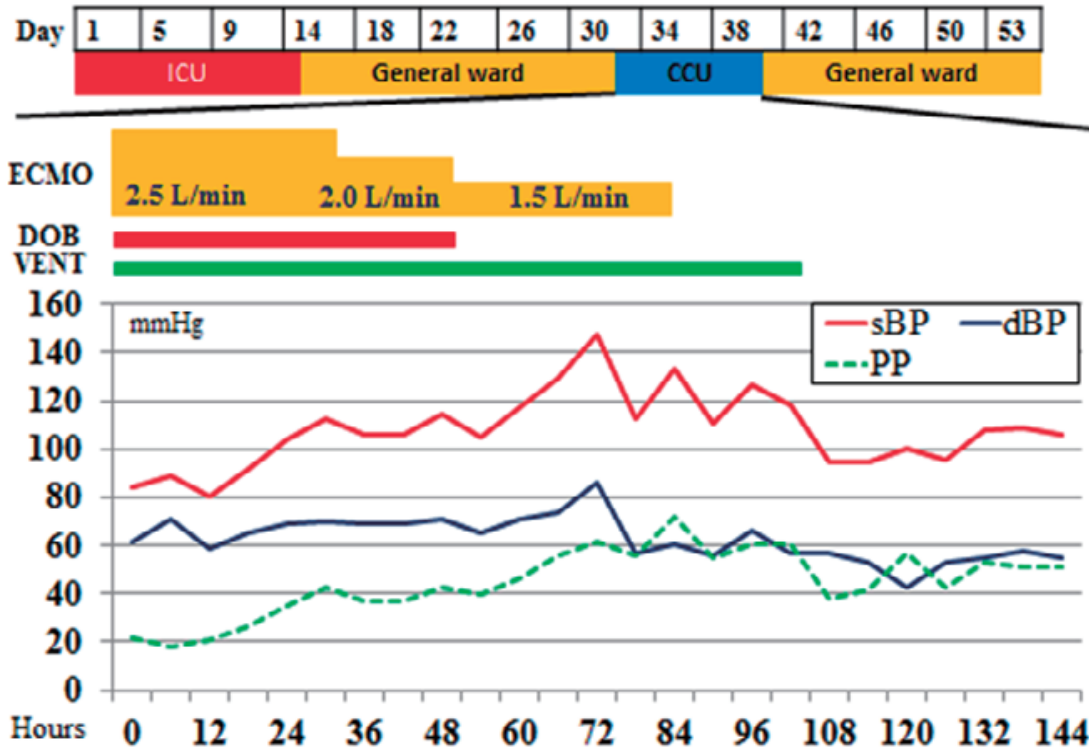

Figure 3. Clinical course. Extracorporeal membrane oxygenation (ECMO) was removed at 72 hours from the readmission to cardiac care unit (CCU). The patient was extubated without significant neurological abnormality at 102 hours from the readmission of CCU. And the patient left the $\mathrm{CCU}$ at 144 hours from the readmission of $\mathrm{CCU}$. 
There are several literatures regarding the combination of V-A ECMO and thrombolysis for massive PE. ${ }^{12,13)}$ As compared to the combination of V-A ECMO and anticoagulation therapy, the combination of V-A ECMO and thrombolytic therapy should have an advantage to reduce thrombotic burden, but it would have a greater risk of bleeding complications. ${ }^{14)}$ Therefore, thrombolytic therapy should be the first line therapy for patients without any bleeding risk. However, since serious bleeding events are frequently observed in patients with V-A ECMO ${ }^{15)}$ additional bleeding caused by thrombolytic therapy can be fatal in patients with a massive PE. Although clinical guidelines recommended percutaneous catheter-directed treatment as an alternative to surgical pulmonary embolectomy for patients whom full dose systemic thrombolysis was contraindicated ${ }^{16}{ }^{16}$ we did not perform catheter-directed therapy, because there was the potential risk of severe complications such as periprocedural hemodynamic deterioration, distal embolization, pulmonary artery perforation, lung hemorrhage, and pericardial tamponade. ${ }^{17}$

The combination of V-A ECMO and conventional anticoagulation has some limitations. Because cannulas for V-A ECMO are large (13.5 Fr for artery and $18 \mathrm{Fr}$ for vein), it would be difficult to use those cannulas for patients with peripheral arterial disease. Furthermore, since $\mathrm{V}$-A ECMO requires anticoagulation to prevent clotting in the system, we cannot use it in patients with contraindication to anticoagulation. In conclusion, the combination of $\mathrm{V}-\mathrm{A}$ ECMO and conventional anticoagulation may be the preferred first line therapy for the patients with cardiogenic shock following a massive PE.

\section{Disclosures}

Consent: Written informed consent was obtained from patient for the publication of this case report.

Conflict of interest: The authors declare no conflicts of interest in association with the present study.

\section{References}

1. Meneveau N. Therapy for acute high-risk pulmonary embolism: thrombolytic therapy and embolectomy. Curr Opin Cardiol 2010; 25: 560-7. (Review)

2. Nasrin S, Cader FA, Salahuddin M, Nazrin T, Shafi MJ. Pulmo- nary embolism with floating right atrial thrombus successfully treated with streptokinase: a case report. BMC Res Notes 2016; 9: 371 .

3. Ozmen C, Deniz A, Akilli RE, et al. Ultrasound accelerated thrombolysis may be an effective and safe treatment modality for intermediate risk/submassive pulmonary embolism. Int Heart J 2016; 57: 91-5.

4. Sádaba JR, Greco E, Alvarez LA, Pulitani I, Juaristi A, Goiti JJ. The surgical option in the management of acute pulmonary embolism. J Card Surg 2008; 23: 729-32.

5. Riedel M. Acute pulmonary embolism 2: treatment. Heart 2001; 85: 351-60. (Review)

6. Swol J, Buchwald D, Ewers A, Schildhauer TA. Arteriovenous extracorporeal membrane oxygenation (ECMO). A therapeutic option for fulminant pulmonary embolism. Med Klin Intensivmed Notfmed (German) 2013; 108: 63-8.

7. Long B, Koyfman A. Current controversies in thrombolytic use in acute pulmonary embolism. J Emerg Med 2016; 51: 37-44.

8. Sharma V, Goldberg HD, Zubkus D, Shears LL, Kaczorowski DJ. Successful management of cardiac arrest due to pulmonary embolus using extracorporeal membrane oxygenation and ultrasound-accelerated catheter-directed thrombolysis. Ann Thorac Surg 2016; 101: e107-9.

9. Li T, Zhang WW, Bai W, Zhai S, Pang Z. Warfarin anticoagulation before angioplasty relieves thrombus burden in Budd-Chiari syndrome caused by inferior vena cava anatomic obstruction. J Vasc Surg 2010; 52: 1242-5.

10. Nakamura M, Nishikawa M, Komuro I, et al. Apixaban for the treatment of Japanese subjects with acute venous thromboembolism (AMPLIFY-J Study). Circ J 2015; 79: 1230-6.

11. Fujino T, Yamazaki Y, Yamazaki A, et al. Efficacy of dabigatran for dissolving deep vein thromboses in outpatients with a deteriorated general condition. Int Heart J 2015; 56: 395-9.

12. Sangalli F, Greco G, Galbiati L, Formica F, Calcinati S, Avalli L. Regional thrombolysis with tenecteplase during extracorporeal membrane oxygenation: a new approach for left ventricular thrombosis. J Card Surg 2015; 30: 541-3.

13. Silvetti S, Pappalardo F, Melisurgo G, et al. Ultrasoundaccelerated thrombolysis and extracorporeal membrane oxygenation in a patient with massive pulmonary embolism and cardiac arrest. Circ Cardiovasc Interv 2013; 6: e34-6.

14. Marti C, John G, Konstantinides S, et al. Systemic thrombolytic therapy for acute pulmonary embolism: a systematic review and meta-analysis. Eur Heart J 2015; 36: 605-14. (Review)

15. Mazzeffi M, Greenwood J, Tanaka K, et al. Bleeding, transfusion, and mortality on extracorporeal life support: ECLS Working Group on Thrombosis and Hemostasis. Ann Thorac Surg 2016; 101: 682-9.

16. Konstantinides SV, Torbicki A, Agnelli G, et al. 2014 ESC guidelines on the diagnosis and management of acute pulmonary embolism. Eur Heart J 2014; 35: 3033-69.

17. Engelberger RP, Kucher N. Catheter-based reperfusion treatment of pulmonary embolism. Circulation 2011; 124: 2139-44. 